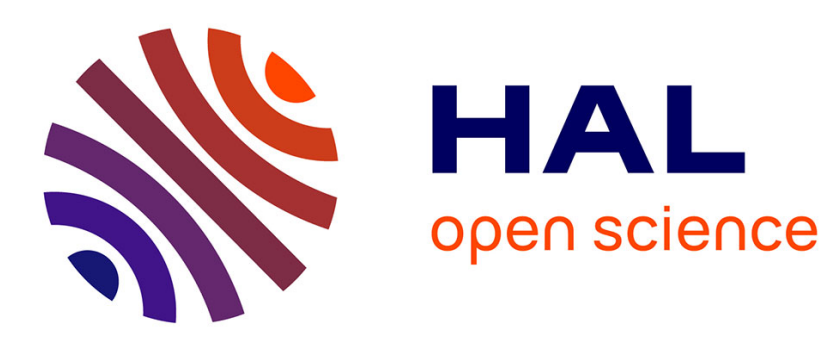

\title{
Formulation and well-posedness of the dynamics of rigid-body systems with perfect unilateral constraints Patrick Ballard
}

\section{To cite this version:}

Patrick Ballard. Formulation and well-posedness of the dynamics of rigid-body systems with perfect unilateral constraints. Philosophical Transactions of the Royal Society A: Mathematical, Physical and Engineering Sciences, 2001, 359 (1789), pp.2327-2346. 10.1098/rsta.2001.0854 . hal-01722467

\section{HAL Id: hal-01722467 \\ https://hal.science/hal-01722467}

Submitted on 4 Mar 2018

HAL is a multi-disciplinary open access archive for the deposit and dissemination of scientific research documents, whether they are published or not. The documents may come from teaching and research institutions in France or abroad, or from public or private research centers.
L'archive ouverte pluridisciplinaire HAL, est destinée au dépôt et à la diffusion de documents scientifiques de niveau recherche, publiés ou non, émanant des établissements d'enseignement et de recherche français ou étrangers, des laboratoires publics ou privés. 


\title{
Formulation and well-posedness of the dynamics of rigid-body systems with perfect unilateral constraints
}

\author{
By PATRICK BALLARD \\ Laboratoire de Mécanique des Solides, Ecole Polytechnique, \\ 91128 Palaiseau Cédex, France (ballard@lms.polytechnique.fr)
}

The classical theory of rigid-body systems dynamics with perfect bilateral constraints is extended in order to take perfect unilateral constraints into account. A systematic formulation of the dynamics is derived and the most general admissible form of the impact-constitutive equation is obtained. Well-posedness of the evolution problem is proved under the assumption that the data are analytic.

Keywords: analytical dynamics; non-smooth mechanics; impact

\section{Introduction}

Rigid-body systems dynamics with perfect bilateral constraints has been well established on firm mathematical foundations for a long time. In this paper, we aim at giving such a status to the dynamics of rigid-body systems with perfect unilateral constraints.

Any mechanical theory relies on a geometric description of the system under study. This is always a schematization. As a consequence, most of the time, the equation of motion has to be completed with some constitutive equation. A constitutive equation conveys the physical information that has been removed by the over-schematization of the geometric description. In fact, the well-posedness of the dynamics generally serves implicitly as a guideline to the identification of the general form of the constitutive law, although thermodynamic considerations can also play an important part.

The dynamics of rigid-body systems with perfect unilateral constraints necessarily involves an impact-constitutive equation. We shall try to identify the most general form of the impact-constitutive equation that is compatible with the well-posedness of the dynamics. Thus, we shall build the theory step by step, justifying the necessity of each hypothesis by a counter-example. These hypotheses will be classified into two categories. Those which convey a physical assumption will be called 'constitutive' hypotheses and the others, whose aim is to prevent mathematical pathologies, will be called 'regularity' hypotheses. Since one aim is to obtain general forms of constitutive laws, one has to make sure that the constitutive laws do not depend on any particular parametrization of the system. For this reason, we are going to try to obtain intrinsic formulations of dynamics, that is, formulations which do not rely on a particular choice for the parametrization of the system. This necessarily requires the use of the language of differential geometry. However, only the most elementary level of differential geometry is needed. 


\section{The dynamics of rigid-body systems}

(a) The geometric assumption: rigidity

Classical mechanics postulates the existence of a three-dimensional oriented affine Euclidean space $\mathcal{E}$, sometimes called the (Galilean) real world, and an absolute chronology represented (after the choice of an origin) by a real number, generally denoted by $t$. The vector space associated with $\mathcal{E}$ will be denoted by $E$.

A solid is represented by its real world reference configuration, which is nothing but a possible geometric locus of all the material points of the solid in $\mathcal{E}$. The geometric assumption of rigidity can be stated as follows: the only real-world configuration of that solid which can be observed are obtained from the real-world reference configuration by direct isometries. Therefore, once the real-world reference configuration has been fixed, any real-world configuration of the solid is represented by a direct isometry $q$. Since any direct isometry on $\mathcal{E}$ can be split into a translation and a rotation, the set of all direct isometries can be identified with $E \times \mathbb{S O} 3$ (where $\mathbb{S O} 3$ denotes the set of all direct orthogonal endomorphisms on $E$, endowed with its standard manifold structure). It is said that $E \times \mathbb{S O} 3$ is the (abstract) configuration manifold of the rigid solid. Since its dimension is 6 , we say that the rigid solid has six degrees of freedom (DOF).

Other idealizations of rigid solids can appear: the infinitely thin rigid bar whose configuration manifold is $E \times \mathbb{S} 2$ ( $\mathbb{S} 2$ denotes the two-dimensional sphere equipped with its standard manifold structure) and the punctual particle whose configuration manifold is simply $E$.

A motion of a rigid solid is a curve on its configuration manifold $Q$ (a mapping from a time-interval $I$ into $Q$ ). The derivative of the motion at instant $t$ is denoted by $\dot{q}(t)$. This is called the abstract (or, sometimes, generalized) velocity and is an element of the tangent bundle $T Q$ of the configuration manifold. One often encounters the name 'state space' for $T Q$, in which case $\dot{q}(t)$ is also called a state of the system.

The mass distribution in the rigid solid is a bounded positive measure on the realworld reference configuration. It allows, classically, the association of any state of the system with its kinetic energy $K(q, \dot{q})$. It defines a positive-definite quadratic form on each tangent space of $Q$, endowing the configuration manifold with a Riemannian structure. This Riemannian metric is naturally called the kinetic metric. From now on, whenever we speak of a configuration manifold, it will always be supposed to be equipped with its Riemannian structure.

A rigid-body system is a finite collection of rigid bodies. The configuration manifold of a rigid-body system is the cross-product $Q_{1} \times Q_{2} \times \cdots \times Q_{n}$ of the individual configuration manifold $Q_{i}$ of each rigid body of the system.

Notation. For $Q$ being a smooth Riemannian manifold of dimension $d$, we shall denote by

(i) $T Q$ and $T^{*} Q$, the tangent and cotangent bundles;

(ii) $\Pi_{Q}$ and $\Pi_{Q}^{*}$, the natural projection mappings of $T Q$ and $T^{*} Q$;

(iii) $\langle\cdot, \cdot\rangle_{q}$, the local duality product between tangent space $T_{q} Q$ and cotangent space $T_{q}^{*} Q$ 
(iv) $(\cdot, \cdot)_{q}$ and $\|\cdot\|_{q}$, the local scalar product and norm on $T_{q} Q$ (a * will be added when referring to the scalar product and norm on $\left.T^{*} Q\right)$;

(v) $b$ (and $\sharp=b^{-1}$, its inverse), the isomorphism of vector bundles from $T Q$ onto $T^{*} Q$ naturally associated with the Riemannian metric of $Q$.

The abstract velocity $\dot{q}(t) \in T Q$ of a motion $q(t)$ will alternatively be denoted by $(q(t), \dot{q}(t))$. This is clearly a redundant notation since the base-point $q=\Pi_{Q}(\dot{q})$ is contained in the derivative, but I believe that this notation will be an aid to understanding. More generally, an element $v$ of $T Q$ will also be denoted by $(q, v)$ with $q$ being the base-point of $v$.

Any (local) chart $\psi$ on the configuration manifold is called a (local) parametrization. For an abstract configuration $q \in Q, \psi(q)$ is an element of $\mathbb{R}^{d}$ that we denote by $\left(q^{1}, q^{2}, \ldots, q^{d}\right)$. Each time a given parametrization will be under consideration, we shall write $q=\left(q^{1}, q^{2}, \ldots, q^{d}\right)$. The natural basis of $T_{q} Q$ (respectively, $T_{q}^{*} Q$ ) naturally associated with the chart $\psi$ is denoted by $\left(e_{1}(q), e_{2}(q), \ldots, e_{d}(q)\right)$ (respectively, $\left.\left(e^{1}(q), e^{2}(q), \ldots, e^{d}(q)\right)\right)$. For $(q, v)$ belonging to $T Q$, we denote by $v^{i}(i=1,2, \ldots, d)$ its components in the natural basis and we shall write

$$
v=v^{i} e_{i}(q) \text {. }
$$

Einstein's summation convention will always apply, unless explicitly stated otherwise. As usual, $g_{i j}(q)$ will be the covariant components of the metric in the considered chart and $g^{i j}(q)$ its contravariant components; $\Gamma_{j k}^{i}(q)$ will be the associated Christoffel symbols:

$$
\Gamma_{j k}^{i}(q)=\frac{1}{2} g^{i h}(q)\left(\frac{\partial g_{h k}}{\partial q^{j}}(q)+\frac{\partial g_{j h}}{\partial q^{k}}(q)-\frac{\partial g_{j k}}{\partial q^{h}}(q)\right) .
$$

For $q(t)$ being a curve on $Q$ and $v$ being a vector field on that curve, the covariant derivative of $v$ along $q(t)$ is denoted by

$$
\frac{\mathrm{D}}{\mathrm{d} t} v(t)=\left(\frac{\mathrm{d}}{\mathrm{d} t} v^{i}(t)+\Gamma_{j k}^{i}(q(t)) v^{j}(t) \dot{q}^{k}(t)\right) e_{i}(q(t)) .
$$

\section{(b) Formulation of the dynamics}

Consider a rigid-body system of configuration manifold $Q$ and a motion $q(t)$ of that system. The power of inertial forces at instant $t$ is, by definition, the time derivative at $t$ of the kinetic energy:

$$
\frac{\mathrm{d}}{\mathrm{d} t} K(q, \dot{q})=\frac{1}{2} \frac{\mathrm{d}}{\mathrm{d} t}(\dot{q}(t), \dot{q}(t))_{q(t)}=\left(\frac{\mathrm{D}}{\mathrm{d} t} \dot{q}(t), \dot{q}(t)\right)_{q(t)}=\left\langle b \frac{\mathrm{D}}{\mathrm{d} t} \dot{q}(t), \dot{q}(t)\right\rangle_{q(t)} .
$$

Hence, it is seen that the power of inertial forces at time $t$ defines the cotangent vector $b \mathrm{D} \dot{q}(t) / \mathrm{d} t \in T_{q(t)}^{*} Q$. An arbitrary element $T_{q} Q$ is often called a virtual velocity of the system in the configuration $q$. Then, the linear form $b \mathrm{D} \dot{q}(t) / \mathrm{d} t$ is called the virtual power of inertial forces.

The analysis of the dynamics has to take into account external and internal efforts. They define a linear form $f \in T_{q}^{*} Q$ on each tangent space of the configuration manifold, which is classically named the virtual power of external and internal efforts. The reason for such a modelling of efforts by duality is that it ensures the consistency 
of the modelling of the efforts with the geometric description of the system. The linear form $f(q, \dot{q} ; t) \in T_{q}^{*} Q$ is allowed to depend not only on time but also on the current state of the system.

The fundamental principle of classical mechanics asserts that the virtual power of inertial forces should equal at every instant the virtual power of external and internal efforts:

$$
\forall t, \quad b \frac{\mathrm{D}}{\mathrm{d} t} \dot{q}(t)=f(q(t), \dot{q}(t), t) .
$$

Equation (2.1) is referred to as the equation of motion. It is a second-order differential equation on the configuration manifold. To express it in a particular parametrization of the system, the following is useful.

Proposition 2.1 (Lagrange). Let $\psi$ be a local chart and $q(t)$ a $C^{2}$ motion on Q. One has

$$
b \frac{\mathrm{D}}{\mathrm{d} t} \dot{q}(t)=\left(\frac{\mathrm{d}}{\mathrm{d} t} \frac{\partial}{\partial \dot{q}^{i}} K(q(t), \dot{q}(t))-\frac{\partial}{\partial q^{i}} K(q(t), \dot{q}(t))\right) e^{i}(q(t)) .
$$

We are given an initial instant $t_{0}$ and an initial state $\left(q_{0}, v_{0}\right) \in T Q$. Then, the evolution problem associated with the dynamics of a rigid-body system is the Cauchy problem, as follows.

Problem I. Find $T>t_{0}$ and $q \in C^{2}\left(\left[t_{0}, T[; Q)\right.\right.$ such that

(i) $\left(q\left(t_{0}\right), \dot{q}\left(t_{0}\right)\right)=\left(q_{0}, v_{0}\right)$,

(ii) $\forall t \in\left[t_{0}, T\left[, b \frac{\mathrm{D}}{\mathrm{d} t} \dot{q}(t)=f(q(t), \dot{q}(t), t)\right.\right.$.

(c) Well-posedness of the dynamics

To study the well-posedness (existence and uniqueness of solution) of problem I, we have to specify regularity assumptions on $Q$ and $f$.

Counter-example 1. Consider the evolution equation

$$
\frac{\mathrm{d}^{2}}{\mathrm{~d} t^{2}} q(t)=6|q(t)|^{1 / 3} \quad(q \in \mathbb{R})
$$

with initial condition $(q(0), \dot{q}(0))=(0,0)$. It is readily checked that the two motions defined on $\mathbb{R}^{+} q(t)=0$ and $q(t)=t^{3}$ provide two distinct solutions.

To get well-posedness, we are led to make further hypotheses. Throughout this paper, we shall distinguish two classes of hypotheses: the constitutive hypotheses and the regularity hypotheses. A constitutive hypothesis is one which conveys physical meaning. A regularity hypothesis conveys no physical meaning and is stated to eliminate mathematical pathologies. The following regularity hypothesis is slightly stronger than necessary.

Regularity hypothesis. The Riemannian configuration manifold is of class $C^{2}$ and the mapping $f: T Q \times \mathbb{R} \rightarrow T^{*} Q$ is of class $C^{1}$. 
It should be pointed out that the first part of this hypothesis is actually no hypothesis at all. The configuration manifold of the three-dimensional rigid solid, of the infinitely thin rigid bar or of the punctual particle, with arbitrary mass distribution is $C^{\infty}$ (or, even more, analytic) Riemannian manifolds. The configuration manifold of a rigid-body system (with no constraints), being a cross-product of such manifolds, can be assumed to have arbitrary regularity. This is not a restriction either on the geometry nor on the mass distribution of the system, but on the class of admissible parametrizations.

Under this regularity assumption, we have the following well-posedness result.

Theorem 2.2 (Cauchy). There exists a unique maximal solution for problem I.

More precisely, theorem 2.2 states that there exists $T_{m}>t_{0}\left(T_{m} \in \mathbb{R} \cup\{+\infty\}\right)$ and $q_{m} \in C^{2}\left(\left[t_{0}, T_{m}[, Q)\right.\right.$, being a solution of problem I such that any other solution of problem I is a restriction of $q_{m}$. Of course, we expect that $T_{m}=+\infty$, in which case the dynamics is said to be eternal. This situation cannot be taken for granted, in general.

Counter-example 2. Consider the evolution equation

$$
\frac{\mathrm{d}^{2}}{\mathrm{~d} t^{2}} q(t)=(\dot{q}(t))^{2}
$$

$(q \in \mathbb{R})$ with initial condition $(q(0), \dot{q}(0))=(0,1)$. It is readily checked that the maximal solution is defined on the interval $[0,1[$.

In the usual cases encountered in mechanics, eternal dynamics is ensured by the following general sufficient condition.

Theorem 2.3. The configuration manifold $Q$ is assumed to be a complete Riemannian manifold (this is no hypothesis in the case of rigid-body system with no constraints). The mapping $f$ is supposed to admit the following estimate,

$$
\|f(q, v ; t)\|_{q}^{*} \leqslant l(t)\left(1+d\left(q, q_{0}\right)+\|v\|_{q}\right),
$$

for all $(q, v) \in T Q$ and almost all $t \in\left[t_{0},+\infty[\right.$, where $d(\cdot, \cdot)$ is the Riemannian distance and $l(t)$ is a (necessarily non-negative) function of $L_{\text {loc }}^{1}(\mathbb{R} ; \mathbb{R})$. Then, the dynamics is eternal: $T_{m}=+\infty$.

The proof of theorem 2.3 relies on Gronwall's lemma.

\section{Perfect bilateral constraints}

A constraint describes a type of effort which is not taken into account by the efforts mapping $f$. Indeed, it is possible to specify (partly) some efforts by their kinematical effects. In general, these kinematical effects leave the associated efforts partly undetermined and we have to add phenomenological assumptions about how the constraint acts through a constitutive law of the constraint.

\section{(a) The geometric description}

A (holonomic) bilateral constraint is a restriction on the admissible motions of the system which is expressed by means of a finite number $n$ of smooth real-valued 
functions $\varphi_{i}$ on the configuration manifold $Q$, defining a set $S$ of admissible configurations:

$$
S=\left\{q \in Q ; \forall i \in\{1,2, \ldots, n\}, \varphi_{i}(q)=0\right\} .
$$

The following hypothesis is usual in this framework.

Regularity hypothesis $\mathbf{I}$. The functions $\varphi_{i}$ are functionally independent, that is, for all $q \in S$, the $\mathrm{d} \varphi_{i}(q)(i \in\{1,2, \ldots, n\})$ are linearly independent in $T^{*} Q$.

A straightforward consequence of this hypothesis is that $S$ is a submanifold of $Q$ of dimension $d-n$. As a result, $S$ inherits a Riemannian structure from $Q$. We shall say that $S$ is the configuration manifold of the constrained system.

\section{(b) Formulation of the dynamics}

The realization of the constraint (3.1) necessarily involves a modification of the equation of motion (2.1). This is done by adding to the virtual power of efforts $f(q, \dot{q} ; t)$ a corrective unknown term $R$ called the virtual power of reaction efforts:

$$
\forall t, \quad b \frac{\mathrm{D}}{\mathrm{d} t} \dot{q}(t)=f(q(t), \dot{q}(t), t)+R(t) .
$$

We might expect $R$ to be determined by the geometric constraint (3.1), but, in general, this does not work. We have to add phenomenological assumptions on the way the constraint acts. This is the constitutive law of the constraint.

Constitutive hypothesis II. The bilateral constraint (3.1) is supposed to be perfect (one also says synonymously ideal), that is, the virtual power of the reaction efforts $R$ vanishes in any virtual velocity compatible with the bilateral constraint:

$$
\forall v \in\left\{v \in T_{q} Q ; \forall i \in\{1,2, \ldots, n\},\left\langle\mathrm{d} \varphi_{i}(q), v\right\rangle_{q}=0\right\} \simeq T S, \quad\langle R, v\rangle_{q}=0 .
$$

Thanks to hypotheses I and II, we can write

$$
R(t)=\sum_{i=1}^{n} \lambda_{i}(t) \mathrm{d} \varphi_{i}(q),
$$

for some real-valued functions $\lambda_{i}$.

Now, we formulate the evolution problem associated with the dynamics of rigidbody systems with perfect bilateral constraints. The initial condition is assumed to be compatible with the realization of the constraint $\left(q_{0}, v_{0}\right) \in T S$.

Problem II. Find $T>t_{0}, q \in C^{2}\left(\left[t_{0}, T[; Q)\right.\right.$ and $n$ functions $\lambda_{i} \in C^{0}\left(\left[t_{0}, T[; \mathbb{R})\right.\right.$ such that

(i) $\left(q\left(t_{0}\right), \dot{q}\left(t_{0}\right)\right)=\left(q_{0}, v_{0}\right)$,

(ii) $\forall t \in\left[t_{0}, T[, q(t) \in S\right.$,

(iii) $\forall t \in\left[t_{0}, T\left[, b \frac{\mathrm{D}_{Q}}{\mathrm{~d} t} \dot{q}(t)=f(q(t), \dot{q}(t), t)+\sum_{i=1}^{n} \lambda_{i}(t) \mathrm{d} \varphi_{i}(q(t))\right.\right.$.

Here, we used the notation $\mathrm{D}_{Q} / \mathrm{d} t$ for the covariant derivative to underline the fact that the covariant derivative is understood with respect to the Riemannian structure of $Q$ (and not to that of $S$ ). 
Let $q$ be a point of $Q, v$ a vector in $T_{q} Q$, and $E$ a subspace of $T_{q} Q$. The orthogonal projection of $v$ on $E$ for the scalar product of $T_{q} Q$, induced by the Riemannian structure of $Q$, is denoted by $\operatorname{Proj}_{q}[v ; E]$. Similarly, $\operatorname{Proj}_{q}^{*}\left[v^{*} ; E^{*}\right]$ denotes the orthogonal projection of the cotangent vector $v^{*}$ on the subspace $E^{*}$ of $T_{q}^{*} Q$. If $q(t)$ is a curve on the Riemannian submanifold $S$ of $Q$ and $v$ a vector field on that curve, then we have (see Chavel 1993, p. 54)

$$
\frac{\mathrm{D}_{S} v}{\mathrm{~d} t}=\operatorname{Proj}_{q}\left[\frac{\mathrm{D}_{Q} v}{\mathrm{~d} t} ; T_{q} S\right] .
$$

Therefore, any solution of problem II is seen to be a solution of the following problem.

Problem II'. Find $T>t_{0}$ and $q \in C^{2}\left(\left[t_{0}, T[; S)\right.\right.$ such that

(i) $\left(q\left(t_{0}\right), \dot{q}\left(t_{0}\right)\right)=\left(q_{0}, v_{0}\right)$,

(ii) $\forall t \in\left[t_{0}, T\left[, b \frac{\mathrm{D}_{S}}{\mathrm{~d} t} \dot{q}(t)=\operatorname{Proj}_{q(t)}^{*}\left[f(q(t), \dot{q}(t) ; t) ; T_{q(t)}^{*} S\right]\right.\right.$.

Reciprocally, any solution of problem $\mathrm{II}^{\prime}$ is readily seen to generate a solution of problem II: the two evolution problems are equivalent.

The linear form (cotangent vector) $\operatorname{Proj}_{q}^{*}\left[f(q, \dot{q} ; t) ; T_{q}^{*} S\right]$ equals the restriction of the linear form $f(q, \dot{q} ; t)$ on the space $T_{q} S$ of virtual velocities compatible with the bilateral constraint. Therefore, it is the virtual power of external and internal efforts in any virtual velocity compatible with the constraint.

\section{(c) Well-posedness of the dynamics}

Problem II' has formally the same structure of problem I. Since problems II' and II are equivalent, the results of $\S 2 c$ give the well-posedness of the dynamics of rigidbody systems with perfect bilateral constraints.

Regularity hypothesis III. The configuration manifold $Q$ and the functions $\varphi_{i}$ are of class $C^{2}$ and the mapping $f: T Q \times \mathbb{R} \rightarrow T^{*} Q$ is of class $C^{1}$.

Proposition 3.1. Problems II and $I I^{\prime}$ have a unique maximal solution $q_{m}$.

The analysis of the eternity of the dynamics is provided by theorem 2.3.

Regularity hypothesis I could seem very restrictive. However, dropping it would lead to difficulties.

Counter-example 3. Consider a rigid homogeneous bar of length $l$. The two extremities of the bar are constrained to remain on a fixed circle of diameter $l$. The two corresponding bilateral constraints are supposed to be perfect. This is a simple occurrence of a bilateral constraint, which does not satisfy regularity hypothesis I. At the initial instant, the bar is at rest. A constant force is applied at the middle point of the bar. This force is directed in the plane of the circle but not along the bar. The reader will be convinced that the corresponding evolution problem II admits no solution.

Since the modelling of a rigid-body system with no constraint or with perfect bilateral constraint leads to the construction of mathematical structures of the same type, we state the following definition. 
Definition 3.2. A simple discrete mechanical system is a pair $(Q, f)$, where

(i) $Q$ is a finite-dimensional smooth Riemannian manifold called the configuration manifold.

(ii) $f: T Q \times \mathbb{R} \rightarrow T^{*} Q$ is a smooth mapping satisfying

$$
\forall(q, v) \in T Q, \quad \forall t \in \mathbb{R}, \quad \Pi_{Q}^{*}(f(q, v ; t))=q,
$$

called the efforts mapping.

\section{Perfect unilateral constraints}

The consideration of elementary examples shows that the dynamics of rigid-body systems can lead to some prediction of the motion where some bodies of the system overlap in the real world. Of course, this should not be allowed. Hence, very often, one has to add the statement of non-penetration conditions to a simple discrete mechanical system. This is a simple occurrence of unilateral constraint. In this section, we shall discuss the consideration of perfect unilateral constraints in simple discrete mechanical systems.

\section{(a) The geometric description}

Consider a simple discrete mechanical system with configuration manifold $Q$. A unilateral constraint is a restriction on the admissible motions of the system, which is expressed by means of a finite number $n$ of smooth real-valued functions $\varphi_{i}$ on the configuration manifold $Q$, so that the set of all admissible configurations $A$ is given by

$$
A=\left\{q \in Q ; \forall i \in\{1,2, \ldots, n\}, \varphi_{i}(q) \leqslant 0\right\} .
$$

The set of all active constraints in the admissible configuration $q \in A$ is defined by

$$
J(q)=\left\{i \in\{1,2, \ldots, n\} ; \varphi_{i}(q)=0\right\} .
$$

The following hypothesis should be compared with regularity hypothesis I of $\S 3 a$.

Regularity hypothesis I. The functions $\varphi_{i}$ are functionally independent in the sense that, for all $q \in A$, the $\mathrm{d} \varphi_{i}(q)(i \in J(q))$ are linearly independent in $T^{*} Q$.

Consider a motion $q(t)$ in $A$ and assume that a right velocity $\dot{q}^{+}(t) \in T_{q(t)} Q$ exists at instant $t$; then $\dot{q}^{+}(t)$ necessarily belongs to the closed convex cone $V(q(t))$ of $T_{q(t)} Q$ defined by

$$
V(q(t))=\left\{v \in T_{q(t)} Q ; \forall i \in J(q(t)),\left\langle\mathrm{d} \varphi_{i}(q(t)), v\right\rangle_{q(t)} \leqslant 0\right\} .
$$

$V(q)$ is called the cone of admissible right velocities at the configuration $q$. In particular,

$$
q \in \AA \text { (i.e. } J(q)=\emptyset) \Longrightarrow V(q)=T_{q} Q .
$$

Similarly, if a left velocity $\dot{q}^{-} \in T_{q} Q$ exists, then $\dot{q}^{-} \in-V(q)$.

\section{(b) Formulation of the dynamics}

The formulation of the dynamics follows Moreau (1983). 
(i) Equation of motion

As for bilateral constraints, the realization of the constraints induces some reaction effort $R$. The following hypotheses are made.

Constitutive hypothesis II. The unilateral constraints are of type contact without adhesion:

$$
\forall v \in V(q), \quad\langle R, v\rangle_{q} \geqslant 0 .
$$

Constitutive hypothesis III. The unilateral constraints are perfect:

$$
\forall v \in\left\{v \in T_{q} Q ; \forall i \in J(q),\left\langle\mathrm{d} \varphi_{i}(q), v\right\rangle_{q}=0\right\}, \quad\langle R, v\rangle_{q}=0 .
$$

As an easy consequence of constitutive hypotheses II and III, we get

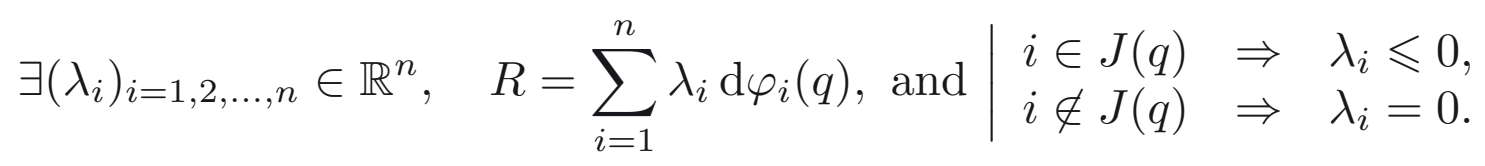

Thus, the reaction effort $R \in T^{*} Q$ must be such that

$$
-R \in N^{*}(q) \stackrel{\text { def }}{=}\left\{\sum_{i=1}^{n} \lambda_{i} \mathrm{~d} \varphi_{i}(q) ; \forall i \in J(q), \lambda_{i} \geqslant 0, \quad \forall i \notin J(q), \lambda_{i}=0\right\} .
$$

$N^{*}(q)$ is a closed convex cone of $T_{q}^{*} Q$ and it is the polar cone of $V(q)$ in the duality $\left(T_{q} Q, T_{q}^{*} Q\right)$, the polar cone of $V(q)$ for the Euclidean structure of $T_{q} Q$ being $N(q)=\sharp\left(N^{*}(q)\right)$.

Now, consider a motion $q(t)$ starting at $q_{0} \in \AA$ at time $t_{0}$ with velocity $v_{0}$. Assumed to be continuous, $q(t)$ remains in $\AA$ on a right neighbourhood of $t_{0}$. By formula (4.2), the reaction effort $R$ vanishes as long as $q(t)$ is in $\AA$ and the motion is governed by the following ordinary differential equation.

$$
\begin{aligned}
\left(q\left(t_{0}\right), \dot{q}\left(t_{0}\right)\right) & =\left(q_{0}, v_{0}\right), \\
b \frac{\mathrm{D} \dot{q}}{\mathrm{~d} t} & =f(q, \dot{q} ; t) .
\end{aligned}
$$

Suppose that the solution of this Cauchy problem meets $\partial A$ at some instant greater than $t_{0}$. Denote by $T$ the smallest of these instants. The motion admits a left velocity vector $v_{T}^{-}$at time $T$. Of course, the following may happen: $v_{T}^{-} \notin V(q(T))$. In this case, no differentiable extension of the motion can exist in $A$ for $t$ greater than $T$. The requirement of differentiability has to be dropped. An instant such as $T$ is called an instant of impact.

However, we are still going to require the existence of a right velocity vector $\dot{q}^{+}(t) \in$ $V(q(t))$ at every instant $t$. The right velocity need not be a continuous function of time and the equation of motion,

$$
b \frac{\mathrm{D} \dot{q}^{+}}{d t}=f\left(q, \dot{q}^{+} ; t\right)+R,
$$

should be understood in the sense of Schwartz's distribution. Actually, we require $R$ to be a vector-valued measure rather than a general distribution.

We denote by $\operatorname{MMA}(I ; Q)$ (motions with measure acceleration) the set of all absolutely continuous motions $q(t)$ from a real interval $I$ to $Q$ admitting a right velocity 
$\dot{q}^{+}(t)$ at every instant $t$ of $I$ and such that the function $\dot{q}^{+}(t)$ has locally bounded variation over $I$. Naturally, bounded variation is classically defined only for functions taking values in a normed vector space. However, for any absolutely continuous curve $q(t)$ on a Riemannian manifold, parallel translation along $q(t)$ classically provides intrinsic identification of the tangent spaces at different points of the curve and, therefore, the definitions can easily be carried over to this case (for the precise mathematical setting, see Ballard (2000)). Any motion $q \in \operatorname{MMA}(I ; Q)$ admits a left and a right velocity, $\dot{q}^{-}$and $\dot{q}^{+}$, in the classical sense at any instant. Moreover, the covariant Stieltjes measure $\mathrm{D} \dot{q}^{+}$of its right velocity $\dot{q}^{+}$is intrinsically associated with any motion $q \in \operatorname{MMA}(I ; Q)$. The equation of motion takes the form,

$$
b \mathrm{D} \dot{q}^{+}=f\left(q, \dot{q}^{+} ; t\right) \mathrm{d} t+R,
$$

where $\mathrm{d} t$ denotes the Lebesgue measure. We have to give a precise meaning to condition (4.2) with $R$ being a vector-valued measure.

Convention. We shall write

$$
R \in-N^{*}(q(t))
$$

to mean that there exist $n$ non-positive real measures $\lambda_{i}$ such that

$$
R=\sum_{i=1}^{n} \lambda_{i} \mathrm{~d} \varphi_{i}(q(t)) \quad \text { and } \quad \forall i \in\{1,2, \ldots, n\}, \quad \operatorname{Supp} \lambda_{i} \subset\left\{t ; \varphi_{i}(q(t))=0\right\} .
$$

Using this convention, the final form of the equation of motion is

$$
R=b \mathrm{D} \dot{q}^{+}-f\left(q(t), \dot{q}^{+}(t) ; t\right) \mathrm{d} t \in-N^{*}(q(t)) .
$$

A straightforward consequence of the equation of motion is that an impact (that is, a discontinuity of the right velocity $\dot{q}^{+}$) can only occur at an instant $t$ such that $J(q(t)) \neq \emptyset$. This fact is a justification for the following definition.

Definition 4.1. An impact occurring at time $t$ is said to be simple if $J(q(t))$ contains exactly one element. If $J(q(t))$ contains at least two elements, the impact is said to be multiple.

\section{(ii) The impact-constitutive equation}

We begin this section with an example. Consider the one-degree-of-freedom mechanical system whose configuration space is $\mathbb{R}$ equipped with its canonical Euclidean structure. The efforts mapping $f$ vanishes identically and the unilateral constraint is represented by the single function $\varphi_{1}(q)=q$ so that the admissible configuration set $A$ is $\mathbb{R}^{-}$. At initial time $t_{0}=0$, we consider an initial state $\left(q_{0}, v_{0}\right)$ such that $q_{0}<0$ and $v_{0}>0$. It is readily seen from the equation of motion (4.4) that an impact necessarily occurs at time $t=-q_{0} / v_{0}$. At this time, the left velocity is $v_{0}$. But, the right velocity can take any negative value and whatever it is, it is compatible with the equation of motion.

The reason for this indetermination lies in the phenomenological nature of the interaction of the system with the obstacle. This missing information has to be added. 
Constitutive hypothesis IV. The interaction of the system with the obstacle at time $t$ is completely determined by the current configuration $q(t)$ and the current left velocity $\dot{q}^{-}(t)$. In other terms, we postulate the existence of a mapping $\mathcal{F}: T Q \rightarrow T Q$ describing the interaction of the system with the obstacle during an impact:

$$
\forall t, \quad \dot{q}^{+}(t)=\mathcal{F}\left(q(t), \dot{q}^{-}(t)\right) .
$$

To ensure compatibility with the equation of motion (4.4), the mapping $\mathcal{F}$ should satisfy

$$
\forall q \in A, \quad \forall v^{-} \in-V(q), \quad \begin{aligned}
& \mathcal{F}\left(q, v^{-}\right) \in V(q), \\
& \mathcal{F}\left(q, v^{-}\right)-v^{-} \in-N(q) .
\end{aligned}
$$

Moreover, we add the assumption that the kinetic energy of the system cannot increase during an impact.

$$
\forall q \in A, \quad \forall v^{-} \in-V(q), \quad\left\|\mathcal{F}\left(q, v^{-}\right)\right\|_{q} \leqslant\left\|v^{-}\right\|_{q} .
$$

Let us comment on hypothesis IV. When two solids hit, their bouncing is actually governed by the propagation of deformation waves in each of the two solids. But, from the very beginning, we have adopted the simple framework in which each solid is supposed to be rigid, that is, for the sake of simplicity, we have chosen not to take into consideration any phenomena relying on the deformation of the solids. Thus, we cannot expect the theory to be able to predict the outcome of an impact experiment. The aim of constitutive hypothesis IV is to introduce the missing information into the theory. Of course, in practical situations, we have to identify the unknown mapping $\mathcal{F}$. This can be done either by means of experiments or by use of a refined theory. For example, the theory of elastodynamics could be used to predict the outcome of an impact in every impact configuration. The result would be an identification of the mapping $\mathcal{F}$. In any case, there is a very large amount of work in precisely identifying $\mathcal{F}$. This is the price we have to pay for describing sophisticated physical phenomena in a very simple framework. Actually, this issue is faced in any mechanical theory (for example, the theory of elasticity). Naturally, in each mechanical theory, the question arises as to what amount of missing constitutive information should be introduced. Most of the time, well-posedness of the resulting evolution problem serves as a guideline to stating the constitutive hypotheses.

Definition 4.2. Equation (4.5), with mapping $\mathcal{F}$ satisfying requirements (4.6) and (4.7) is called the impact-constitutive equation. An impact-constitutive equation which ensures the conservation of kinetic energy during an impact,

$$
\forall q \in A, \quad \forall v^{-} \in-V(q), \quad\left\|\mathcal{F}\left(q, v^{-}\right)\right\|_{q}=\left\|v^{-}\right\|_{q},
$$

is called elastic.

There always exist many mappings $\mathcal{F}$ satisfying requirements (4.6) and (4.7).

Example 4.3. Let $e: T Q \rightarrow[0,1]$ be an arbitrary function. The mapping $\mathcal{F}$ defined by

$$
\mathcal{F}\left(q, v^{-}\right)=\operatorname{Proj}_{q}\left[v^{-} ; V(q)\right]-e\left(q, v^{-}\right) \operatorname{Proj}_{q}\left[v^{-} ; N(q)\right],
$$

is easily seen to satisfy requirements (4.6) and (4.7). The associated impact-constitutive equation is often called the canonical impact-constitutive equation. It is elastic if and only if $e \equiv 1$. The function $e$ is classically called the restitution coefficient. 
(a)

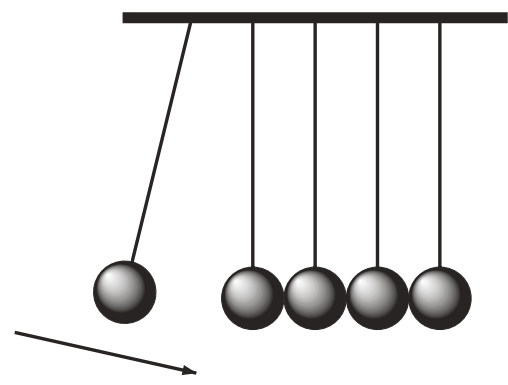

(b)

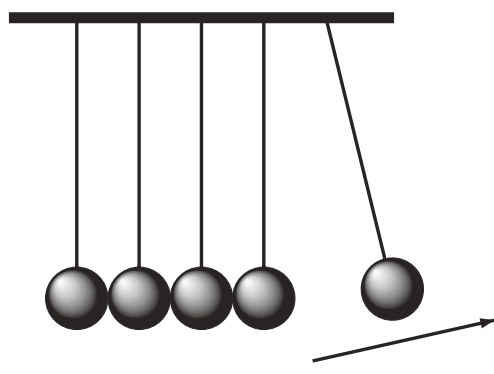

(c)

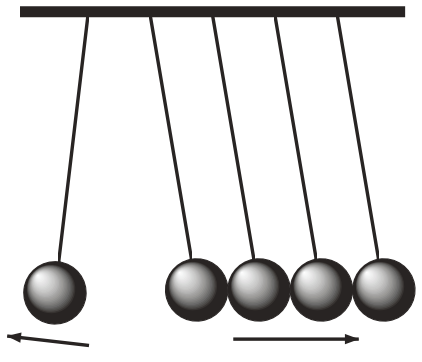

Figure 1. Newton's cradle.

The reason why the canonical impact-constitutive equation is distinguished is that, in situations where only simple impacts can occur (for example, if the unilateral constraint is represented by a single function $\varphi_{1}$ ), then the impact-constitutive equation must be the canonical one (this is a simple consequence of requirements (4.6) and (4.7)). However, in the case of multiple impacts, the canonical impact-constitutive equation has no specific physical relevance. A simple occurrence of a multiple impact is provided by Newton's cradle. The principle of the experiment is sketched in figure $1 a$. Its outcome is sketched in figure $1 b$. It should be compared with the prediction of the canonical elastic impact-constitutive equation, which is sketched in figure $1 c$.

The following proposition is a straightforward and useful consequence of requirements (4.6) and (4.7).

Proposition 4.4. Let $\mathcal{F}$ be a constitutive mapping satisfying requirements (4.6) and (4.7). Then, we have

$$
\forall q \in A, \quad \forall v^{-} \in V(q) \cap(-V(q)), \quad \mathcal{F}\left(q, v^{-}\right)=v^{-} .
$$

We conclude this section by a comment on requirement (4.7). At first glance, it could seem to be unnecessary. The following counter-example proves that if it were omitted, then uniqueness of solution for the resulting evolution problem would surely not hold.

Counter-example 4. Consider the one-degree-of-freedom discrete mechanical system whose configuration space is $\mathbb{R}$ equipped with its canonical Euclidean structure. The efforts mapping is supposed to be constant: $f(q, \dot{q} ; t) \equiv 2$. To this simple discrete mechanical system we add the unilateral constraint described by the single function $\varphi_{1}(q)=q$. Thus, $A=\mathbb{R}^{-}$. The impact-constitutive equation is given by formula (4.8), where the restitution coefficient is supposed to be the constant $1 / 2$ : $e\left(q, \dot{q}^{-}\right) \equiv 1 / 2$. This mechanical system is a formal description of the physical occurrence of a single particle subjected to gravity and bouncing on the floor. Consider the initial instant $t_{0}=0$ and the initial state $\left(q_{0}, v_{0}\right)=(-1,0)$. It is readily seen that the function $q: \mathbb{R}^{+} \rightarrow \mathbb{R}^{-}$defined by

$$
\begin{array}{lll}
\forall t & \in[0,1], & q(t)=t^{2}-1, \\
\forall t \in\left[3-\frac{1}{2^{n-1}}, 3-\frac{1}{2^{n}}\right], & q(t)=t^{2}+\left(-6+\frac{3}{2^{n}}\right) t+\left(3-\frac{1}{2^{n-1}}\right)\left(3-\frac{1}{2^{n}}\right), \\
\forall t \in[3,+\infty[, & q(t)=0,
\end{array}
$$

$(n \in \mathbb{N})$ belongs to $\operatorname{MMA}\left(\mathbb{R}^{+} ; \mathbb{R}^{-}\right)$and satisfies

(i) the initial condition, 


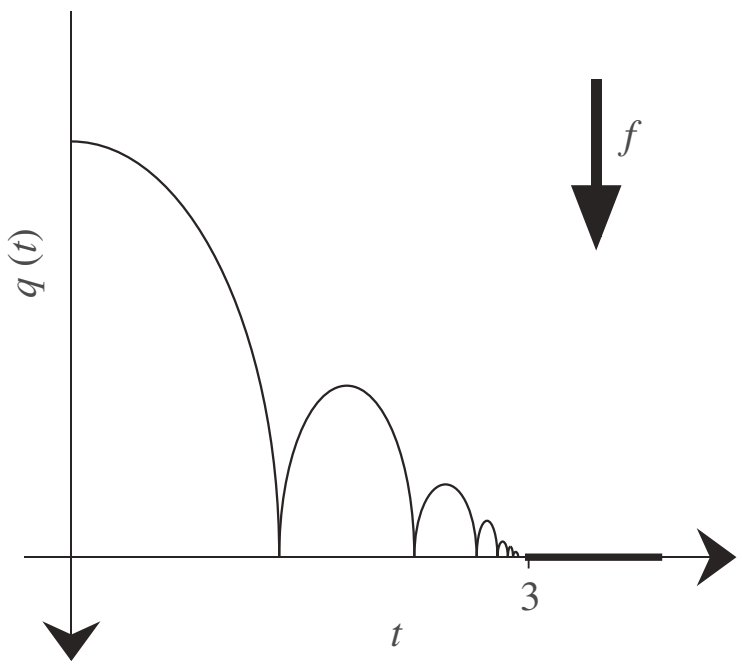

Figure 2. Motion of a punctual particle subjected to gravity and bouncing on the floor.

(ii) the equation of motion (4.4) (with $f(q, \dot{q} ; t) \equiv 2)$,

(iii) the impact-constitutive equation (4.8) (with $e(q, \dot{q}) \equiv 1 / 2)$.

This motion is pictured in figure 2. Note, by the way, that it exhibits an infinite number of impacts on a compact time subinterval. It could easily be proved that no motion, defined on $[0,+\infty$ [, with finite number of impacts on every compact interval can exist.

Now, we are going to analyse what happens when the flow of time is reversed. Define $q^{\prime}$ by

$$
q^{\prime}\left\{\begin{aligned}
{[0,4] } & \rightarrow \mathbb{R}^{-} \\
t & \mapsto q(4-t) .
\end{aligned}\right.
$$

Considering the initial state $\left(q_{0}, v_{0}\right)=(0,0)$ at $t_{0}=0$, it is easily seen that $q^{\prime}$ satisfies

(i) that initial condition,

(ii) the equation of motion (4.4) (with $f(q, \dot{q} ; t) \equiv 2$ ),

(iii) the impact-constitutive equation (4.8) (with $e(q, \dot{q}) \equiv 2$ ).

However, $q^{\prime \prime} \equiv 0$ is also seen to satisfy the same initial condition, equation of motion and impact-constitutive equation. This example demonstrates that we cannot expect uniqueness of solution when adopting the canonical impact-constitutive equation (4.8) with restitution coefficient $e \equiv 2$ (or any real number strictly greater than 1). However, the canonical impact-constitutive equation with a restitution coefficient strictly greater than 1 violates requirement (4.7).

\section{(iii) The evolution problem I}

Now, we formulate the evolution problem associated with the dynamics of rigidbody systems with perfect bilateral and unilateral constraints. The initial condition is assumed to be compatible with the realization of the constraint $v_{0} \in V\left(q_{0}\right)$.

Problem III. Find $T>t_{0}$ and $q \in \operatorname{MMA}\left(\left[t_{0}, T[; Q)\right.\right.$ such that

(i) $\left(q\left(t_{0}\right), \dot{q}^{+}\left(t_{0}\right)\right)=\left(q_{0}, v_{0}\right)$, 
(ii) $\forall t \in\left[t_{0}, T[, q(t) \in A\right.$,

(ii) $R \stackrel{\text { def }}{=} b \mathrm{D} \dot{q}^{+}-f\left(q(t), \dot{q}^{+}(t) ; t\right) \mathrm{d} t \in-N^{*}(q(t))$,

(iv) $\left.\forall t \in] t_{0}, T\right], \dot{q}^{+}(t)=\mathcal{F}\left(q(t), \dot{q}^{-}(t)\right)$.

The equation of motion is understood in the sense of convention (4.3) and the impactconstitutive equation is supposed to fulfil requirements (4.6) and (4.7).

No regularity assumption has yet been made on the mapping $f$. This will be done in the next section, where the well-posedness of problem III is studied. However, we can infer from $\S 2 c$ that $f$ will be assumed to be at least of class $C^{1}$. We can state an elementary property of any solution (if there are any) of problem III.

Proposition 4.5 (Energy inequality). Any solution $(T, q)$ of problem III satisfies

$$
\begin{aligned}
& \forall t_{1}, t_{2} \in\left[t_{0}, T\left[, \quad t_{1} \leqslant t_{2},\right.\right. \\
& \qquad \begin{aligned}
K\left(q\left(t_{2}\right), \dot{q}^{+}\left(t_{2}\right)\right)-K\left(q\left(t_{1}\right), \dot{q}^{+}\left(t_{1}\right)\right) & =\frac{1}{2}\left\|\dot{q}^{+}\left(t_{2}\right)\right\|_{q\left(t_{2}\right)}^{2}-\frac{1}{2}\left\|\dot{q}^{+}\left(t_{1}\right)\right\|_{q\left(t_{1}\right)}^{2} \\
& \leqslant \int_{t_{1}}^{t_{2}}\left\langle f\left(q(s), \dot{q}^{+}(s) ; s\right), \dot{q}^{+}(s)\right\rangle_{q(s)} \mathrm{d} s .
\end{aligned}
\end{aligned}
$$

Proof. Since

$$
\begin{aligned}
\frac{1}{2}\left\|\dot{q}^{+}\left(t_{2}\right)\right\|_{q\left(t_{2}\right)}^{2}- & \frac{1}{2}\left\|\dot{q}^{+}\left(t_{1}\right)\right\|_{q\left(t_{1}\right)}^{2}= \\
& \int_{t_{1}}^{t_{2}}\left\langle\dot{q}^{+}(t), f\left(q(t), \dot{q}^{+}(t) ; t\right)\right\rangle_{q(t)} \mathrm{d} t+\int_{] t_{1}, t_{2}\right]}\left\langle\frac{\dot{q}^{+}+\dot{q}^{-}}{2}, R\right\rangle_{q},
\end{aligned}
$$

we have only to prove that the last integral is non-positive. Set

$$
\left.\left.D=\{t \in] t_{1}, t_{2}\right] ; \dot{q}^{-}(t) \neq \dot{q}^{+}(t)\right\} .
$$

$D$ is (at most) countable and, therefore, Lebesgue-negligible. On the one hand, we have

$$
\int_{] t_{1}, t_{2}\right] \backslash D}\left\langle\frac{\dot{q}^{+}+\dot{q}^{-}}{2}, R\right\rangle_{q}=\int_{] t_{1}, t_{2}\right] \backslash D}\left\langle\dot{q}^{+}, R\right\rangle_{q}=\int_{] t_{1}, t_{2}\right] \backslash D}\left\langle\dot{q}^{-}, R\right\rangle_{q},
$$

where the second integral is non-negative by convention (4.3), whereas the third integral is non-positive. As a consequence, the three integrals vanish. On the other hand,

$$
\begin{aligned}
\int_{D}\left\langle\frac{\dot{q}^{+}(t)+\dot{q}^{-}(t)}{2}, R\right\rangle_{q(t)} & =\int_{D}\left(\frac{\dot{q}^{+}(t)+\dot{q}^{-}(t)}{2}, \mathrm{D} \dot{q}^{+}\right)_{q(t)} \\
& =\frac{1}{2} \sum_{t \in D}\left(\left\|\dot{q}^{+}(t)\right\|_{q(t)}^{2}-\left\|\dot{q}^{-}(t)\right\|_{q(t)}^{2}\right)
\end{aligned}
$$

which is non-positive by virtue of requirement (4.7). 


\section{(c) Well-posedness of the dynamics}

To study the well-posedness of problem III, we need to state regularity assumptions on the data. Looking at those of $\S 3 c$, we could expect to be able to prove the wellposedness of problem III under the assumption that the functions $\varphi_{i}$ and the mapping $f$ are of class $C^{2}$ and $C^{1}$, respectively. The following counter-example originally due to Bressan (1960) and Schatzman (1978) shows that uniqueness does not hold in general even if the data are supposed to be of class $C^{\infty}$.

Counter-example 5. Consider a simple discrete mechanical system whose configuration space is $\mathbb{R}$, equipped with its canonical Euclidean structure. This is the configuration space of a particle with unit mass constrained to move along a line. A fixed obstacle at the origin is taken into consideration. It gives rise to a unilateral constraint described by the single function:

$$
\varphi_{1}(q)=q .
$$

Therefore, the admissible configuration set is $A=\mathbb{R}^{-}$. The impact-constitutive equation is supposed to be elastic. Here, the geometry is so poor that this statement determines completely the impact-constitutive equation. It is necessarily the canonical one with restitution coefficient $e \equiv 1$. The efforts mapping $f$ is supposed not to depend on the state but only on time. It will be denoted by $f(t)$. The initial instant is $t_{0}=0$ and the initial state is $\left(q_{0}, v_{0}\right)=(0,0)$. The corresponding problem III admits here the following simple formulation.

Find $T>0$ and $q \in \operatorname{MMA}([0, T[; \mathbb{R})$ such that

(i) $\left(q(0), \dot{q}^{+}(0)\right)=(0,0)$,

(ii) $\forall t \in[0, T[, q(t) \leqslant 0$,

(iii) $R \stackrel{\text { def }}{=} \mathrm{d} \dot{q}^{+}-f(t) \mathrm{d} t$ is a non-positive real measure such that

$$
\operatorname{Supp} R \subset\{t \in[0, T[; q(t)=0\},
$$

(iv) $\forall t \in] 0, T\left[, \quad\left\{\begin{array}{l}q(t) \neq 0 \Rightarrow \dot{q}^{+}(t)=\dot{q}^{-}(t), \\ q(t)=0 \Rightarrow \dot{q}^{+}(t)=-\dot{q}^{-}(t) .\end{array}\right.\right.$

Here $\mathrm{d} \dot{q}^{+}$is merely the classical Stieltjes measure associated with the function with locally bounded variation $\dot{q}^{+}$. We investigate uniqueness under the assumption that $f$ is of class $C^{\infty}$ and non-negative:

$$
\forall t \in \mathbb{R}^{+}, \quad f(t) \geqslant 0 .
$$

Then, it is readily seen that the null function $\tilde{q} \equiv 0$ on $\mathbb{R}^{+}$is a solution of that problem, whatever is the non-negative $C^{\infty}$ function $f$. Now, we are going to construct an explicit example of such a function $f$ in such a way that the associated evolution problem III admits another solution, distinct from the identically vanishing one.

First, define a Massin function $\rho$ by

$$
\rho\left\{\begin{array}{l}
\mathbb{R} \rightarrow \mathbb{R} \\
x \mapsto \mid\left\{\begin{array}{l}
0 \quad \text { if } x \in]-\infty, 0] \cup[1,+\infty[, \\
\left.C \mathrm{e}^{1 / x(x-1)} \text { if } x \in\right] 0,1[,
\end{array}\right.
\end{array}\right.
$$


where $C$ is a real constant which is chosen to get $\int_{-\infty}^{+\infty} \rho(x) \mathrm{d} x=1$. Define

$$
T=\sum_{n=0}^{\infty} \frac{(n+5)^{2}}{(n+1)(n+2)(n+3)(n+4)},
$$

and, for every $n \in \mathbb{N}$,

$$
\begin{aligned}
a_{n} & =\sum_{i=n}^{\infty} \frac{(i+5)^{2}}{(i+1)(i+2)(i+3)(i+4)} \\
\delta_{n} & =\frac{n+5}{(n+1)(n+2)(n+4)} \quad\left(\text { i.e. } \delta_{n}=\frac{n+3}{n+5}\left(a_{n}-a_{n+1}\right)<a_{n}-a_{n+1}\right), \\
f_{n} & =\frac{1}{n !} \\
v_{n} & =-\frac{1}{(n+3) !} .
\end{aligned}
$$

Now, the functions $f(t)$ and $v(t)$, from $[0, T[$ to $\mathbb{R}$, are defined by

$$
\begin{aligned}
& f(0)=0, \\
& f(t)= \begin{cases}0, & \text { if } t \in\left[a_{n+1}, a_{n+1}+\delta_{n}[,\right. \\
\frac{1}{2} f_{n} \rho\left(\frac{t-a_{n+1}-\delta_{n}}{a_{n}-a_{n+1}-\delta_{n}}\right), & \text { if } t \in\left[a_{n+1}+\delta_{n}, a_{n}[,\right.\end{cases}
\end{aligned}
$$

and

$$
\begin{aligned}
& v(0)=0, \\
& v(t)= \begin{cases}v_{n+1}, & \text { if } t \in\left[a_{n+1}, a_{n+1}+\delta_{n}[,\right. \\
v_{n+1}+\frac{1}{2} f_{n} \int_{a_{n+1}+\delta_{n}}^{t} \rho\left(\frac{s-a_{n+1}-\delta_{n}}{a_{n}-a_{n+1}-\delta_{n}}\right) \mathrm{d} s, & \text { if } t \in\left[a_{n+1}+\delta_{n}, a_{n}[.\right.\end{cases}
\end{aligned}
$$

Finally, the function $q:[0, T[\rightarrow \mathbb{R}$ is defined by

$$
q(t)=\int_{0}^{t} v(s) \mathrm{d} s .
$$

The graph of the functions $f(t)$ and $q(t)$ is sketched in figure 3 . The reader will easily check that

(i) $f(t)$ is a $C^{\infty}$ non-negative function on $[0, T[$,

(ii) $(T, q)$ is a solution of the considered evolution problem,

(iii) the only instants at which $q(t)=0$ are 0 and the $a_{n}$.

Therefore, $q$ and $\tilde{q}$ provide two solutions of the evolution problem. These two solutions do not coincide on any open subinterval of $[0, T[$. Therefore, uniqueness of solution for problem III cannot be asserted, even in the case where the data are supposed to be of class $C^{\infty}$. Percivale (1985) was the first to notice that, in the above example, if $f(t)$ is supposed to be analytic, then uniqueness of solution does hold. Recently, a complete 


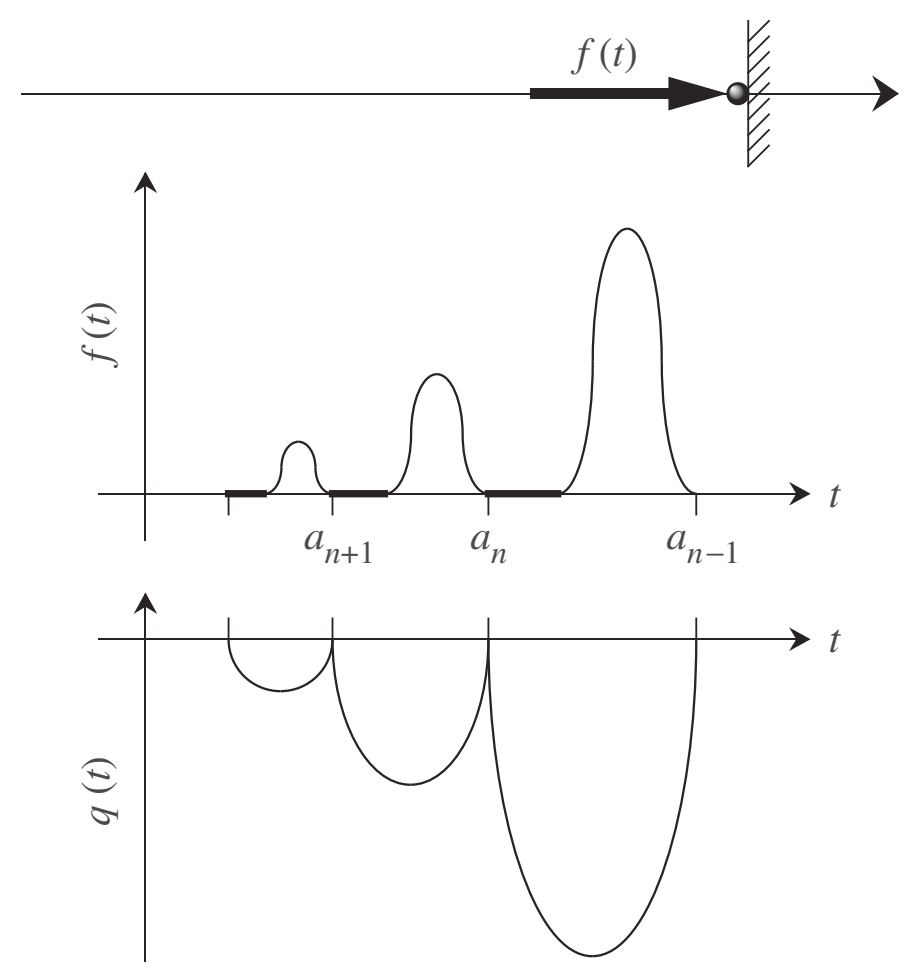

Figure 3. Bressan-Schatzman counter-example.

discussion of the one-degree-of freedom problem was obtained by Schatzman (1998). The general case is treated in Ballard (2000) and is now recalled. Let us just mention that prior existence results had been obtained, but they were limited to the case where the unilateral constraint is represented by a single function (see Schatzman 1978; Monteiro Marques 1993).

Regularity hypothesis V. The Riemannian configuration manifold, the functions $\varphi_{i}$ and the mapping $f: T Q \times \mathbb{R} \rightarrow T^{*} Q$ are analytic.

The proof of the following proposition can be found in Ballard (2000). An earlier proof can also be found in Lötstedt (1982).

Proposition 4.6. Let $q_{0} \in A$ and $v_{0} \in V\left(q_{0}\right)$. Then, there exist $T_{\mathrm{a}}>t_{0}$, an analytic curve $q_{\mathrm{a}}:\left[t_{0}, T_{\mathrm{a}}\left[\rightarrow Q\right.\right.$ and $n$ analytic functions $\lambda_{\mathrm{a} i}:\left[t_{0}, T_{\mathrm{a}}[\rightarrow \mathbb{R}\right.$ such that

(i) $\left(q_{\mathrm{a}}(0), \dot{q}_{\mathrm{a}}^{+}(0)\right)=\left(q_{0}, v_{0}\right)$,

(ii) $\forall t \in\left[t_{0}, T_{\mathrm{a}}\left[, b \frac{\mathrm{D}}{\mathrm{d} t} \dot{q}_{\mathrm{a}}(t)=f\left(q_{\mathrm{a}}(t), \dot{q}_{\mathrm{a}}(t) ; t\right)+\sum_{i=1}^{n} \lambda_{\mathrm{a} i}(t) \mathrm{d} \varphi_{i}\left(q_{\mathrm{a}}(t)\right)\right.\right.$,

(iii) $\forall t \in\left[t_{0}, T_{\mathrm{a}}\left[, \forall i=1,2, \ldots, n, \lambda_{\mathrm{a} i}(t) \leqslant 0, \varphi_{i}\left(q_{\mathrm{a}}(t)\right) \leqslant 0, \lambda_{\mathrm{a} i}(t) \varphi_{i}\left(q_{\mathrm{a}}(t)\right)=0\right.\right.$.

Moreover, the solution of this evolution problem is unique in the sense that any other analytic solution $\left(T, q, \lambda_{1}, \ldots, \lambda_{n}\right)$ is either a restriction or analytic extension of $\left(T_{\mathrm{a}}, q_{\mathrm{a}}, \lambda_{\mathrm{a} 1}, \ldots, \lambda_{\mathrm{a} n}\right)$.

Corollary 4.7. There exists an analytic solution $\left(T_{\mathrm{a}}, q_{\mathrm{a}}\right)$ for problem III.

Proof. Consider the motion $q_{\mathrm{a}}$ furnished by proposition 4.6. It obviously satisfies the initial condition, the unilateral constraint and the equation of motion. The only 
thing which remains to be proven is that it satisfies the impact-constitutive equation. Since $q_{\mathrm{a}}$ is analytic and satisfies the unilateral constraint, we have

$$
\forall t \in] t_{0}, T_{\mathrm{a}}\left[, \quad \dot{q}_{\mathrm{a}}^{-}(t)=\dot{q}_{\mathrm{a}}^{+}(t) \in V\left(q_{\mathrm{a}}(t)\right) \cap\left(-V\left(q_{\mathrm{a}}(t)\right)\right),\right.
$$

and, therefore,

$$
\forall t \in] t_{0}, T_{\mathrm{a}}\left[, \quad \dot{q}_{\mathrm{a}}^{+}(t)=\dot{q}_{\mathrm{a}}^{-}(t)=\mathcal{F}\left(q_{\mathrm{a}}(t), \dot{q}_{\mathrm{a}}^{-}(t)\right),\right.
$$

by proposition 4.4 .

Naturally, the analytic solution furnished by corollary 4.7 will cease to exist at the first instant of impact. This is the reason why we have considered the wider class MMA, which contains motions that are not differentiable in the classical sense. Considering motions in MMA will allow us to extend the solution beyond the first instant of impact. But we must make sure that admitting the wider class of solutions MMA will not introduce parasitic solutions. This is the aim of the following theorem.

Theorem 4.8. Let $\left(T_{\mathrm{a}}, q_{\mathrm{a}}\right)$ be the solution for problem III furnished by corollary 4.7, and $(T, q)$ be an arbitrary solution for problem III. Then, there exists a real number $T_{0}\left(t_{0}<T_{0} \leqslant \min \left\{T_{\mathrm{a}}, T\right\}\right)$ such that

$$
q_{\mid\left[t_{0}, T_{0}[\right.} \equiv q_{\mathrm{a} \mid\left[t_{0}, T_{0}[\right.} \cdot
$$

In other words, there is local uniqueness for problem III.

Local uniqueness is the difficult part in the study of the well-posedness of problem III. A detailed proof of the local uniqueness theorem 4.8 can be found in Ballard (2000). It is written in the framework of the canonical impact-constitutive equation (4.8), but careful examination of the proof shows that the canonical impactconstitutive equation is only used through the energy inequality (proposition 4.5). Since the energy inequality holds for any impact-constitutive equation satisfying requirements (4.6) and (4.7), so does local uniqueness.

Corollary 4.9. There exists a unique maximal solution for problem III.

It was noticed above that the analytical solution for problem III furnished by corollary 4.7 stops existing at the first instant of impact. To overcome this fact, we have proved that local uniqueness still holds in the wider class of motion MMA which allows impacts. But this does not guarantee that the maximal solution for problem III is not going to stop to exist at finite time for unphysical reasons. In other terms, we still do not know if the class MMA is wide enough. Actually, it is wide enough as shown by the following theorem which should be brought aside theorem 2.3.

Theorem 4.10. The configuration manifold $Q$ is assumed to be a complete Riemannian manifold and the mapping $f$ is supposed to admit the following estimate,

$$
\|f(q, v ; t)\|_{q}^{*} \leqslant l(t)\left(1+d\left(q, q_{0}\right)+\|v\|_{q}\right),
$$

for all $(q, v) \in T Q$ and almost all $t \in\left[t_{0},+\infty[\right.$, where $d(\cdot, \cdot)$ is the Riemannian distance and $l(t)$, a (necessarily non-negative) function of $L_{\text {loc }}^{1}(\mathbb{R} ; \mathbb{R})$.

Then, the dynamics is eternal, that is, the maximal solution for problem III is defined on $\left[t_{0},+\infty[\right.$.

For detailed proof the reader is referred to Ballard (2000). Here also, the impactconstitutive equation is only used through the energy inequality. 


\section{(d) Comments}

It is readily seen that the function $q$ displayed in counter-example 4 is the unique maximal solution of problem III corresponding to the situation under consideration. This solution exhibits an accumulation of impacts on the left side of instant $t=3$. However, as predicted by corollary 4.7 , for each instant $t \in \mathbb{R}^{+}$, there exists a right neighbourhood $[t, t+\eta[$ of $t$, such that the restriction of $q$ to $[t, t+\eta[$ is analytic. A straightforward and general consequence of this is the following.

Proposition 4.11. Let $q$ be the maximal solution of problem III furnished by corollary 4.9. Although infinitely many impacts can accumulate at the left of a given instant, such an accumulation of impacts can never occur at the right of any instant. Moreover, in the particular case where the impact-constitutive equation is elastic, the instants of impact are isolated and therefore in finite number in any compact interval of time.

Proof. Since for each instant $t \in\left[t_{0}, T\right.$, there exists a right neighbourhood $[t, t+\eta[$ of $t$, such that the restriction of $q$ to $[t, t+\eta[$ is analytic, we get the first part of the proposition. For the second part, let $\tau$ be an arbitrary instant in $] t_{0}, T[$ and consider the problem III associated with the initial condition $\left(q(\tau),-\dot{q}^{-}(\tau)\right)$, the elastic constitutive impact equation and the effort mapping $g(q, v ; t)$ defined by

$$
g(q, v ; t)=f(q,-v ; \tau-t),
$$

which is analytic. By theorem 4.8, there exists an analytic function $q_{\mathrm{a}}:\left[0, T_{\mathrm{a}}[\rightarrow Q\right.$ which is a solution of this problem III. Any other solution of problem III coincides with $q_{\mathrm{a}}$ on a right neighbourhood of $t=0$. Actually, as seen in the proof of local uniqueness (theorem 4.8), a little bit more is proved: any function $q^{\prime} \in$ $M M A([0, T[; Q)$ satisfying the initial condition, the unilateral constraint, the equation of motion (4.4) and the energy inequality (proposition 4.5) has to coincide with $q_{\mathrm{a}}$ on a right neighbourhood of $t=0$. But, it is readily seen that the function defined by

$$
q^{\prime}(t)=q(\tau-t), \quad t \in\left[0, \tau-t_{0}[,\right.
$$

fulfil these requirements. Thus, $q^{\prime}$ cannot have right accumulation of impacts at $t=\tau$ and, therefore, $q$ cannot have left accumulation of impacts at $t=\tau$ and the instants of impact are isolated. Of course, if $q$ is the maximal solution defined on $\left[t_{0}, T[\right.$, impacts can still accumulate at the left of $T$, as seen in simple examples.

The fact that infinitely many impacts can accumulate at the left of a given instant but not at the right is a specific feature of the analytical setting that is lost in the $C^{\infty}$ setting as seen in counter-example 5. Actually, this counter-example shows that pathologies of non-uniqueness in the $C^{\infty}$ setting are intimately connected to the possibility of right accumulations of impacts. The fact that the analytical setting prevents such right accumulations is the fundamental reason why we can prove uniqueness in this case.

\section{References}

Ballard, P. 2000 The dynamics of discrete mechanical systems with perfect unilateral constraints. Arch. Ration. Mech. Analysis 154, 199-274. 
Bressan, A. 1960 Incompatibilità dei teoremi di esistenza e di unicità del moto per un tipo molto comune e regolare di sistemi meccanici. Annli Scuola Norm. Superiore Pisa III 14, 333-348.

Chavel, I. 1993 Riemannian geometry: a modern introduction. Cambridge University Press.

Lötstedt, P. 1982 Mechanical systems of rigid bodies subject to unilateral constraints. SIAM J. Appl. Math. 42, 281-296.

Monteiro Marques, M. D. P. 1993 Differential inclusions in nonsmooth mechanical problems. Birkhaüser.

Moreau, J. J. 1983 Standard inelastic shocks and the dynamics of unilateral constraints. In Unilateral problems in structural analysis (ed. G. Del Piero \& F. Maceri), pp. 173-221. Springer.

Percivale, D. 1985 Uniqueness in the elastic bounce problem. I. J. Diff. Eqns 56, 206-215.

Schatzman, M. 1978 A class of nonlinear differential equations of second order in time. Nonlin. Analysis 2, 355-373.

Schatzman, M. 1998 Uniqueness and continuous dependence on data for one dimensional impact problems. Math. Computat. Model. 28, 1-18. 